\title{
Efficient Selection and Classification of Infrared Excess Emission Stars Based on AKARI and 2MASS Data
}

\author{
Ya-Fang Huang, Jin-Zeng Li, Jing-hua Yuan and Hong-Li Liu \\ ${ }^{1}$ National Astronomical Observatories, Chinese Academy of Sciences, \\ 20A Datun Road, Chaoyang District, Beijing 100012, China \\ email: huangyf@nao.cas.cn
}

\begin{abstract}
The selection of young stellar objects (YSOs) based on excess emission in the infrared is easily contaminated by post-main-sequence stars and various types of emission line stars with similar properties. We define in this paper stringent criteria for an efficient selection and classification of stellar sources with infrared excess emission based on combined Two Micron All Sky Survey (2MASS) and AKARI colors.
\end{abstract}

Keywords. stars: pre-main sequence - stars: variables: T Tauri, Herbig Ae/Be

\section{Main-sequence Stars and Major Contaminants}

First of all, bright dwarfs and giants with known spectral types were selected from the Hipparcos Catalogue and cross-identified with the 2MASS and AKARI Point Source Catalogues to produce the main-sequence and the post-main-sequence tracks (Figure 1(a)). Several kinds of contaminants were then removed based on their distribution in the color-color (C-C) diagrams.

\section{Young Stellar Objects}

Test sample of Herbig Ae/Be stars and classical T Tauri stars were cross-identified with

Table 1. Source selection criteria defined for various types of YSOs and CBe based on the $\mathrm{C}-\mathrm{C}$ diagrams compiled based on 2MASS and AKARI.

\begin{tabular}{|c|c|}
\hline Source & Defination of the Source Selection Criteria \\
\hline HAeBe & 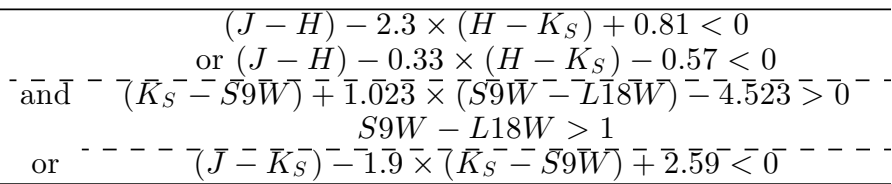 \\
\hline CTTS & 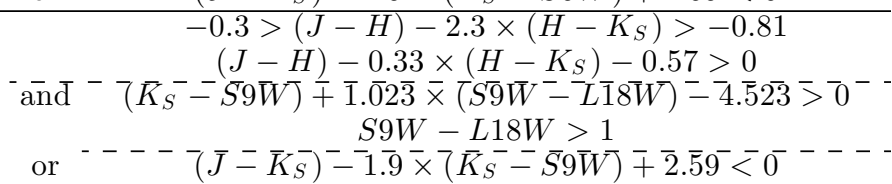 \\
\hline Class I & $\begin{array}{c}\text { and }-{ }^{-}(J-H)-2.3 \times\left(H-K_{S}\right)+0.31<0 \\
-S 9 \bar{W}) \overline{+} 1 . \overline{0} 2 \overline{3} \times \overline{\times}\left(S \overline{9} \bar{W}^{-}-L \overline{1} 8 \bar{W}\right)^{-}-\overline{7} . \overline{0} 46>0^{-}- \\
S 9 W-L 18 W>2\end{array}$ \\
\hline Class II & 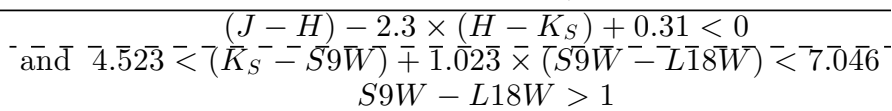 \\
\hline $\mathrm{CBe}$ & 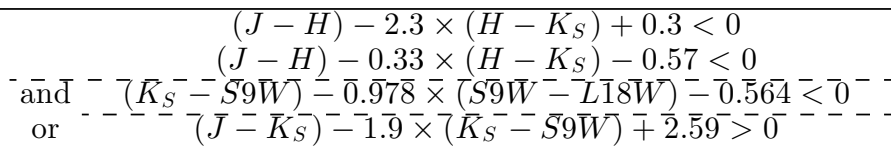 \\
\hline
\end{tabular}




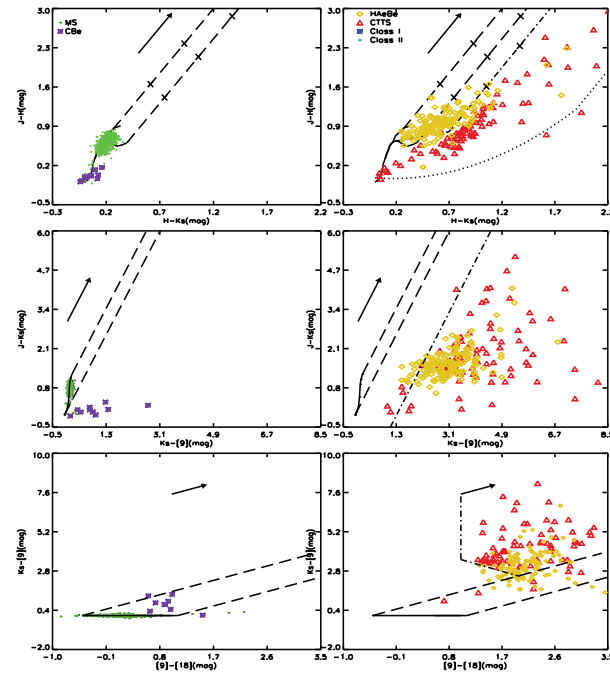

(a)

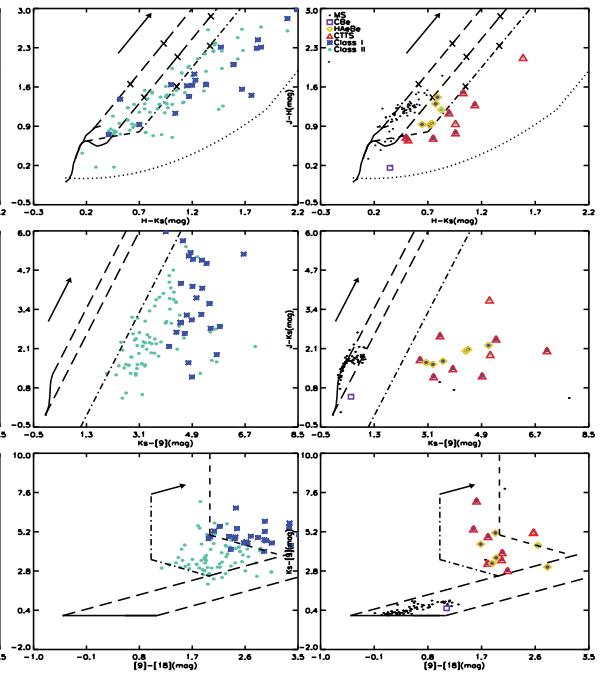

(c)

Figure 1. (a) The distribution of the MS and post-MS sources in C-C diagrams. The tracks of them shown in solid lines were compiled from high-precision data from the Hipparcos Main Catalogue. The symbols in the three diagrams are the same. Green dots and purple asterisk represent normal stars and $\mathrm{CBe}$, respectively. Long dashed lines delineate the reddening band for normal stars. The arrow shows a reddening vector of $A_{v}=5$ mag Rieke \& Lebofsky(1985) in $J H K_{S}$ and $A_{v}=10 \mathrm{mag}$ in $J K_{S}[9]$ and $K_{S}[9][18]$. (b) The distribution of the first test sample of HAeBe stars Thé et al.(1994), and CTTSs from Herbig-Bell Catalogue (left), and the second test sample of Class I and Class II sources from Gutermuth et al.(2008), Gutermuth et al.(2009) (right) in C-C diagrams. Dot-dashed line, short dashed line and dotted line in $J H K_{S}$ indicate the division line between HAeBe and CTTS, the locus of dereddened TTS Meyer et al.(1997) and the locus of dereddened HAeBe Lada \& Adams(1992), respectively. Dot-dashed lines in mid-IR C-C diagrams indicate the left boundary of YSOs. Short-dashed line in $K_{S}$ [9][18] diagram is the division line between Class I and Class II sources. Crosses were over plotted with an interval corresponding to $5 \mathrm{mag}$ of visual extinction in $J H K_{S}$ diagram. The other lines and arrows are the same as those defined in Figure 1(a). (c) A blind classification of the candidate YSOs with excess emission in the IR toward IC 1396. The lines and arrows are the same as those defined in Figure 1(a).

the 2MASS and AKARI catalogs to define the loci of YSOs with different masses on the C-C diagrams(Figure 1(b)). Well classified Class I and Class II sources were taken as a second test sample to discriminate between various types of YSOs at possibly different evolutionary stages. This helped to define the loci of different types of YSOs and a set of criteria for selecting YSOs based on their colors in the near- and mid-infrared. And then our selection and classification of excess emission sources were summarized in Table 1.

\section{Verification of the Sample Selection Criteria}

Candidate YSOs toward IC 1396 indicating excess emission in the near-infrared were employed to verify the validity of the new source selection criteria defined based on C-C diagrams compiled with the 2MASS and AKARI data (Figure 1(c)).

\section{References}

Gutermuth, R. A., Myers, P. C., Megeath, S. T., et al. 2008, , ApJ 674, 336

Gutermuth, R. A., Megeath, S. T., Myers, P. C., et al. 2009, ApJS, 184, 18

Lada, C. J. \& Adams, F. C. 1992, ApJ, 393, 278

Meyer, M. R., Calvet, N., \& Hillenbrand, L. A. 1997, AJ, 114, 288

Rieke, G. H. \& Lebofsky, M. J. 1985, ApJ, 288, 618

Thé, P. S., de Winter, D., \& Pérez, M. R. 1994, A\&AS, 104, 315 